Clinical Vistas

\section{Widened mediastinum in a child with severe trauma}

A 3-year-old girl was struck by a vehicle travelling at a high speed and was thrown approximately $6 \mathrm{~m}$. Emergency medical services staff noted that the patient was seizing with left-deviated eyes. She was rushed to the closest community hospital for resuscitation and was subsequently transferred to our regional trauma centre.

Despite administration of about $55 \mathrm{~mL}$ of crystalloid per kilogram body weight, the patient was tachycardic and hypotensive for her age. A trauma blood panel was drawn, and noncrossmatched blood was transfused. Laboratory test results suggested occult bleeding and myocardial contusion (hemoglobin concentration 8I [normal IIO-I60] g/L, hematocrit 0.24 [normal $0.33^{-0.48}$ ] and troponin I 0.39 [nor$\mathrm{mal}<0.08] \mu \mathrm{g} / \mathrm{L})$.

A chest radiograph taken with the patient in a supine position showed a widened mediastinum with loss of the aortic knob contour (Figure I). Although a frank apical cap or hemothorax was absent, a small amount of fluid was present in the left pleural apex. The left main bronchus was in normal anatomic position. A normal chest radiograph with the patient in an upright position had been taken several months before the trauma and was available for comparison (Figure 2).

Once the patient's condition was stabilized, contrast-enhanced computed tomography was performed and showed a normal aorta with no evidence of hematoma (Figure 3). The thymus accounted for the apparent widened mediastinum seen on the chest radiograph. Intracranial bleeding, identified on a computed tomography scan of her head, accounted for the blood loss. The patient survived and is currently managed at a neurorehabilitation centre.

A widened mediastinum in trauma patients should be considered an aortic injury until proven otherwise. In children, traumatic aortic injuries are uncommon (in about 0.1\% of cases) because of the relative elasticity of their great vessels compared with those in adults. When aortic injury occurs in a child it is most commonly seen at the level of the ligamentum arteriosus and is usually associated with severe deceleration forces. ${ }^{1}$ The signs of aortic injury on a chest radiograph include mediastinal widening, loss of the aortic knob contour, depression of the left main bronchus, deviation of the trachea or esophagus, left pleural cap, hemothorax and fracture of the first or second rib. ${ }^{1,2}$

As illustrated in this case, interpretation of the medistinal outline on a chest radiograph in younger children in the supine position is complicated by the presence of the thymus. ${ }^{3}$ A chest radiograph taken with the child in an upright position can be obtained to clarify the medistinal borders; however, placing a trauma patient with unknown injuries in such a position poses an unacceptable risk. Although arch aortography remains the "gold standard," contrast-enhanced computed tomography and transesophageal echocardiography are acceptable methods to exclude aortic injuries. ${ }^{1-3}$ Computed tomography scans of the chest have the added advantage of delineating the thymic outline. $^{1}$
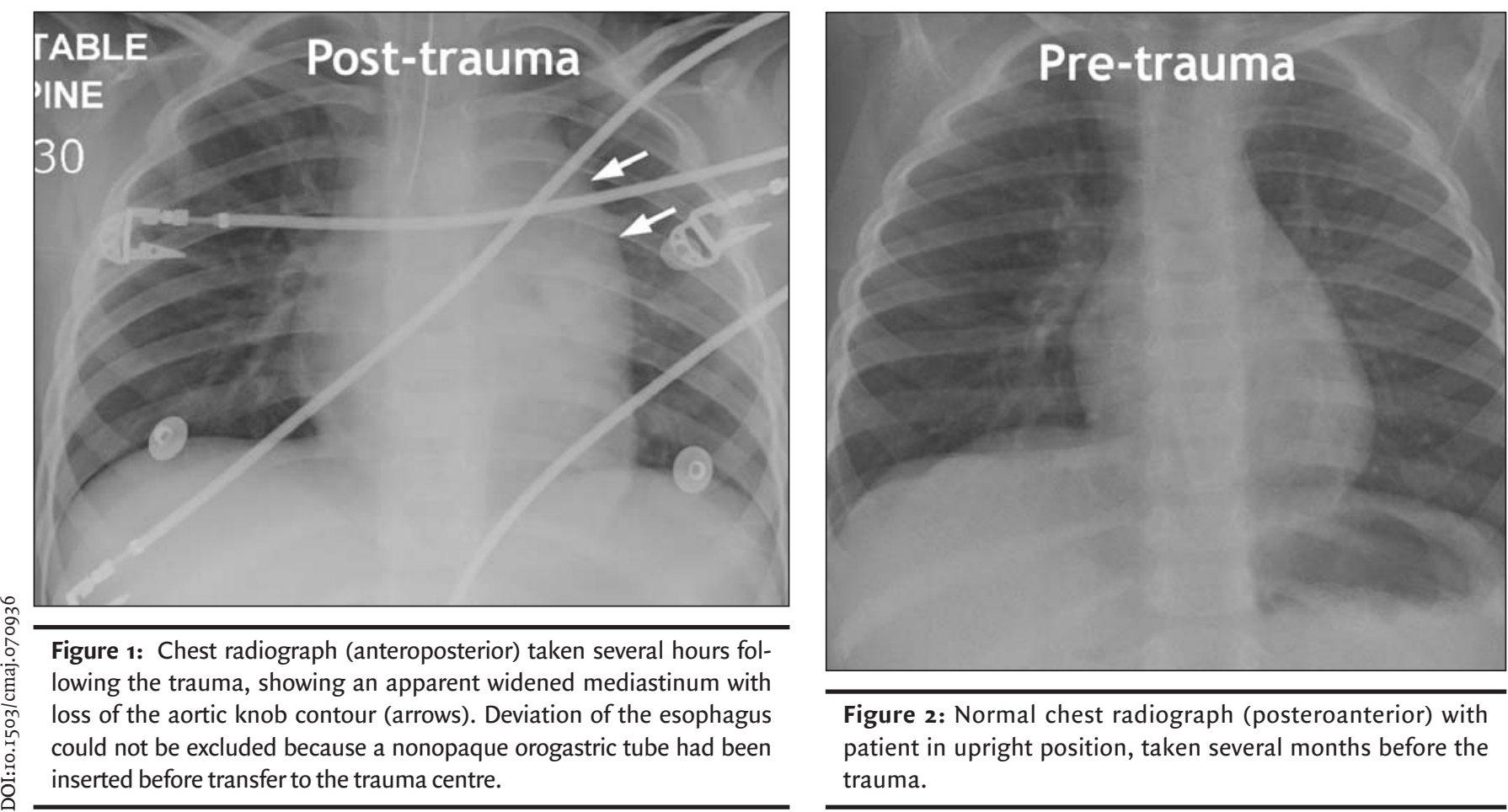

Figure 2: Normal chest radiograph (posteroanterior) with patient in upright position, taken several months before the trauma. 


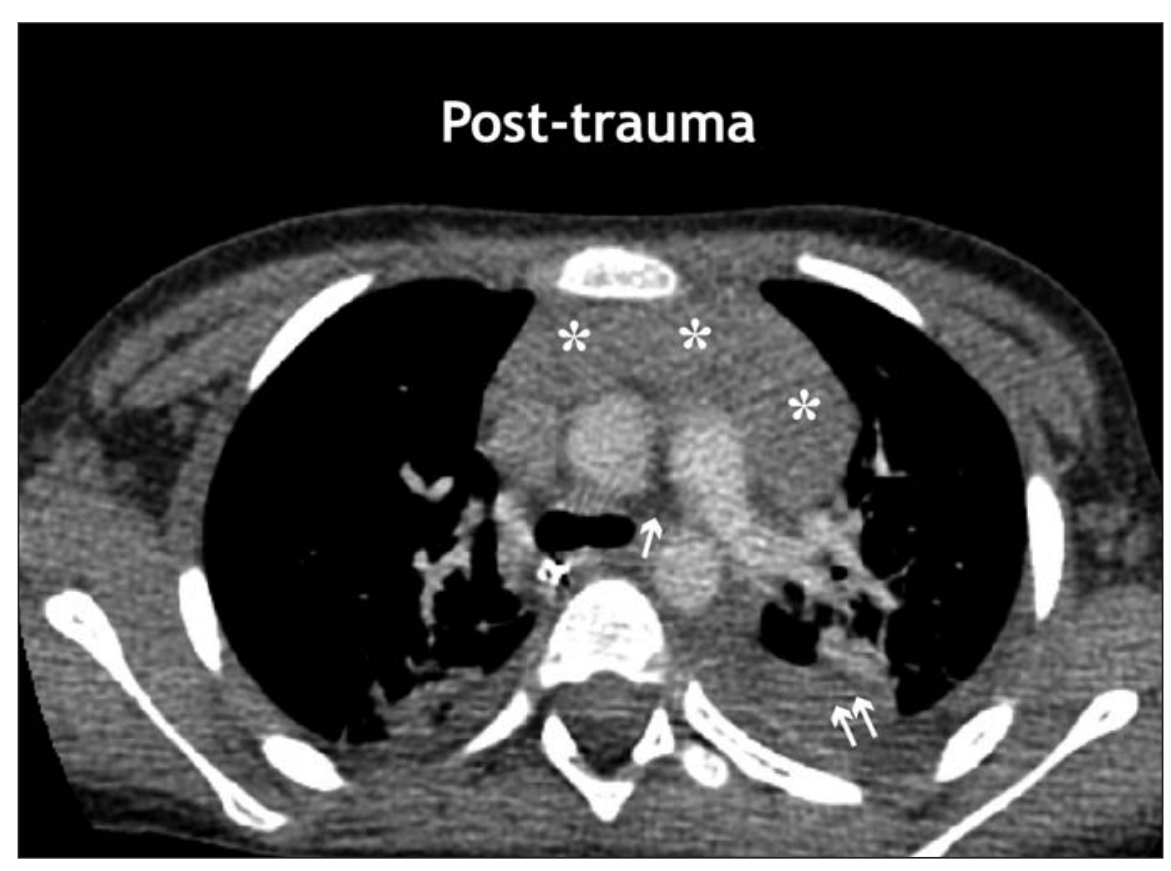

Figure 3: Computed tomography scan, showing normal mediastinum with no evidence of aortic filling defects or pseudoaneurysm. A large thymus (asterisks) consistent with age is evident. A mediastinal fat density (arrow) and pleural effusion (double arrow) are present.
Ilan Buffo-Sequeira MD

Division of Cardiology

Department of Paediatrics

University of Western Ontario

Children's Health Research Institute

London, Ont.

Douglas D. Fraser MD PhD

Division of Critical Care Medicine

Department of Paediatrics

University of Western Ontario

Children's Health Research Institute

and Centre for Critical Illness

Research

London, Ont.

This article has been peer reviewed.

Competing interests: None declared.

\section{REFERENCES}

I. Bliss D, Silen M. Pediatric thoracic trauma. Crit Care Med 2002;30:S409-I5.

2. Stafford PW, Blinman TA, Nance ML. Practical points in evaluation and resuscitation of the injured child. Surg Clin North Am 2002;82:273-30I.

3. Mirvis SE, Shanmuganathan K, editors. Imaging in trauma and critical care. 2nd ed. Philadelphia: Saunders/Elsevier Science; 2003. p. 7II-24.



